\title{
Orbital Prefrontal Cortex Is Required for Object-in-Place Scene Memory But Not Performance of a Strategy Implementation Task
}

\author{
Mark G. Baxter, David Gaffan, Diana A. Kyriazis, and Anna S. Mitchell \\ Department of Experimental Psychology, Oxford University, Oxford OX1 3UD, United Kingdom
}

\begin{abstract}
The orbital prefrontal cortex is thought to be involved in behavioral flexibility in primates, and human neuroimaging studies have identified orbital prefrontal activation during episodic memory encoding. The goal of the present study was to ascertain whether deficits in strategy implementation and episodic memory that occur after ablation of the entire prefrontal cortex can be ascribed to damage to the orbital prefrontal cortex. Rhesus monkeys were preoperatively trained on two behavioral tasks, the performance of both of which is severely impaired by the disconnection of frontal cortex from inferotemporal cortex. In the strategy implementation task, monkeys were required to learn about two categories of objects, each associated with a different strategy that had to be performed to obtain food reward. The different strategies had to be applied flexibly to optimize the rate of reward delivery. In the scene memory task, monkeys learned 20 new object-in-place discrimination problems in each session. Monkeys were tested on both tasks before and after bilateral ablation of orbital prefrontal cortex. These lesions impaired new scene learning but had no effect on strategy implementation. This finding supports a role for the orbital prefrontal cortex in memory but places limits on the involvement of orbital prefrontal cortex in the representation and implementation of behavioral goals and strategies.
\end{abstract}

Key words: macaque; frontal; rule; learning; category; episodic

\section{Introduction}

The orbital prefrontal cortex is well placed to occupy an important role in visual learning. It is densely interconnected with inferotemporal cortex (Webster et al., 1994; Carmichael and Price, 1995; Kondo et al., 2005). Single-unit recordings from orbital prefrontal cortex reveal neuronal firing associated with stimulus and reward value (Watanabe, 1996; Roesch and Olson, 2004). Lesions of orbital prefrontal cortex in macaques impair relearning of a preoperatively acquired nonmatching-to-sample rule, visual recognition memory, reversal of object discrimination problems, extinction of instrumental responding, and goaldirected behavior in a reinforcer devaluation paradigm but spare object-reward association learning (Meunier et al., 1997; Izquierdo et al., 2004; Izquierdo and Murray, 2005). These findings suggest a role for orbital prefrontal cortex in both visual memory and cognitive flexibility. Neuroimaging and neuropsychological studies in humans support this conclusion (Fellows and Farah, 2003; Hornak et al., 2004; Remijnse et al., 2005).

The goal of the present investigation was to establish the extent to which orbital prefrontal cortex plays a role in cognitive flexibility

Received April 22, 2007; revised Aug. 21, 2007; accepted Aug. 31, 2007.

This work was supported by the Wellcome Trust (M.G.B.) and the Medical Research Council (D.G.). We thank M. Brown, G. Daubney, K. Murphy, S. Mygdal, and P. Taylor for technical support and P. Browning and Y. Chudasama for helpful comments on this manuscript.

Correspondence should be addressed to Dr. Mark G. Baxter, Department of Experimental Psychology, Oxford University, South Parks Road, Oxford OX1 3UD, UK.

DOI:10.1523/JNEUROSCI.3369-07.2007

Copyright $\odot 2007$ Society for Neuroscience $\quad 0270-6474 / 07 / 2711327-07 \$ 15.00 / 0$ associated with the choice and application of behavioral strategies used to obtain rewards, rather than simple stimulus-reward contingencies. We trained monkeys on a strategy implementation task in which different categories of objects are associated with different strategies for obtaining reward (Gaffan et al., 2002). Efficient performance on this task requires monitoring recent choice history, continuously switching between categories of objects and using feedback on performance in the form of rewards to guide behavior, as well as inhibiting responding to objects that do not form part of the currently active strategy. If the orbital prefrontal cortex is critical for cognitive flexibility and flexible application of behavioral strategies, or inhibitory control generally, then lesions of orbital prefrontal cortex should impair performance of this task. Performance of this task requires intrahemispheric interaction between frontal and inferotemporal cortex (Gaffan et al., 2002).

We also tested whether removal of orbital prefrontal cortex impaired learning in a scene memory task that models several features of human episodic memory (Gaffan, 1994). This task is reliably impaired by damage to the fornix in monkeys (Gaffan, 1994; Gaffan and Parker, 1996; Parker and Gaffan, 1997) and humans (Aggleton et al., 2000). Learning of new object-in-place scene problems is disrupted by disconnection of the frontal cortex (Browning et al., 2005) or ventrolateral prefrontal cortex (Wilson et al., 2007) from inferotemporal cortex. Additionally, this task relies on discrimination learning and does not require the application of a memory-dependent performance rule like nonmatching-to-sample. This allowed us to assess whether orbital prefrontal cortex also contributes to memory function in 
scene learning, as may be predicted from functional imaging studies of human episodic memory encoding (Frey and Petrides, 2000, 2002), and provided a comparison for effects of orbital prefrontal lesions on the strategy implementation task.

\section{Materials and Methods}

Subjects. Seven rhesus monkeys (Macaca mulatta), five males (CON1, CON2, CON4, ORB1, ORB2) and two females (CON3, ORB3), 3.59$7.44 \mathrm{~kg}(28-51$ months old) at the beginning of behavioral training, participated in this study. The monkeys were housed socially in troops, separated by sex, in indoor enclosures attached to standard caging. Water was always available ad libitum in the home enclosure; each monkey's daily food ration was delivered in the test box and was supplemented with fruit and forage mix in the home enclosure. Four monkeys (CON1, CON2, ORB1, ORB2) underwent pretraining and learned several two-choice visual discrimination problems in a touchscreen apparatus (Baxter and Gaffan, 2007) before beginning training on the strategy implementation task; they then acquired the scene memory task. The other three monkeys (CON3, CON4, ORB3) underwent pretraining, then learned the scene memory task followed by the strategy implementation task.

At the completion of preoperative training and a preoperative performance test on strategy implementation and scene learning, three monkeys (ORB1, ORB2, ORB3) received bilateral ablations of orbital prefrontal cortex, and four (CON1, CON2, CON3, CON4) were retained as unoperated controls. The preoperative and postoperative performance tests were identical for all seven monkeys. Although all comparisons for these tasks could be made on a within-subjects basis, the presence of unoperated controls confirmed that the concurrent testing in multiple behavioral tasks produced stable measures of performance. The unoperated controls also served as a comparison for an additional test of object-reward association learning performed in a between-subjects design after the completion of postoperative testing in strategy implementation and scene learning.

Apparatus. Behavioral testing took place in an automated apparatus. Each monkey was taken from the home enclosure into the test cubicle in a wheeled transport cage, which was fixed in front of a video-display unit with a touch-sensitive screen $(380 \times 280 \mathrm{~mm}, 800 \times 600$ pixel resolution). The monkey could reach through horizontally oriented bars $(\sim 45$ $\mathrm{mm}$ apart) at the front of the cage to reach the screen and the rewards. Stimulus presentation, recording of touches to the screen, and reward delivery were all under computer control. A pellet dispenser delivered $190 \mathrm{mg}$ of banana-flavored or sugar pellets (P. J. Noyes, Lancaster, $\mathrm{NH}$ ) into a food cup located below the touchscreen. Pellet delivery produced a click from the pellet dispenser as well as a $500 \mathrm{~ms}$ tone from the computer. A metal "lunchbox" $(\sim 200 \times 100 \times 100 \mathrm{~mm})$ was located to the left of the food cup and was filled with a mixture of wet monkey chow, seeds, apple, banana, orange, nuts, and dates. Infrared cameras positioned at different locations within the test cubicle permitted observation of the monkey while it was performing the task. The entire apparatus was located in an experimental cubicle that was dark except for the illumination of the video screen.

Behavioral testing: pretraining. The monkeys that had experience with a discrimination learning task in the touchscreen (Baxter and Gaffan, 2007) had no additional pretraining before beginning training on the strategy implementation task (described in the next section). The remaining monkeys were shaped to enter the transport cage from their home enclosure, and once they were reliably taking food in the test cubicle, pretraining began. First, reward pellets were delivered on a variable-interval ( $2 \mathrm{~min}$ ) schedule to accustom them to take pellets in the test box. After several days of pellet training, the touchscreen was activated and the screen was filled with an array of different-colored alphanumeric characters on a black background (in a different size and typeface than those used in the main task). Touches to any location on the screen resulted in pellet delivery. In the third stage, single alphanumeric characters were presented in random locations on the screen and remained until touched; a touch caused the character to disappear and a reward pellet to be delivered. Gradually, the complexity of the display was increased by introducing additional visual elements (a colored background, colored ellipse segments, and a single large alphanumeric character). When monkeys were reliably completing 50 trials in a single test session with minimal accuracy errors (i.e., touching any location on the screen other than the small alphanumeric character), they began training on the scene memory task. The monkeys with discrimination learning experience underwent this third stage of pretraining between acquisition of the strategy task and the scene task.

Strategy implementation task. This task is identical to that described by Gaffan et al. (2002), except that clip-art stimuli were used instead of compound alphanumeric characters (supplemental Fig. 1, available at www.jneurosci.org as supplemental material). The strategy implementation task required monkeys to learn about two categories of objects. Each category was associated with a different strategy that had to be performed to obtain food reward, deemed "persistent" and "sporadic." Efficient performance of the task required alternation of choices between persistent and sporadic objects, with the switch occurring when reward had been earned for selection of one category. Monkeys learned the task using four pairs of objects, each pair containing one item from each of the two categories. These four pairs of objects were used throughout all preoperative and postoperative testing.

A pair of objects appeared on the touchscreen on each trial, containing one object from each category, and the monkey was allowed to choose one of the two objects. The left-right position of the objects on the screen was randomized across trials. After one of the two objects was touched, the screen blanked for a $5 \mathrm{~s}$ intertrial interval before the next trial was presented. Monkeys could earn rewards in one of two ways. First, four consecutive choices of the persistent object within each pair resulted in delivery of a reward pellet after the fourth persistent choice. Second, any time after receiving a reward for choosing four persistent objects in a row, a single choice of an object from the second category (sporadic) resulted in pellet delivery, but another sporadic reward was not given until another persistent reward had been earned. Thus, monkeys were required to alternate between choices of persistent and sporadic objects and had to execute different behavioral strategies to obtain rewards from the objects in the two categories. The dependent measure was the trials/reward ratio. The choice sequence that would optimize the rate of reward delivery was for the monkey to choose the persistent object in the pair on four consecutive trials, then the sporadic object on the following trial, and then to repeat this sequence of choices, resulting in two rewards for every five trials (a trials/reward ratio of 2.5). Failing to choose the sporadic object immediately after receiving a reward for choosing four persistent objects in a row, interrupting chains of persistent responses with choices of sporadic objects, or continuing to choose the sporadic object before another reward had been earned for choosing persistent objects all contributed to less-than-optimal performance and an elevation of the trials/ reward ratio. In each test session, monkeys chose objects across trials until they had earned 50 rewards.

Training procedures were identical to those of Gaffan et al. (2002) and proceeded in five phases. Briefly, monkeys were trained on this task by presenting one pair of objects at a time (containing one persistent object and one sporadic object) until the trials/reward ratio was 2.94 or lower in each of two consecutive sessions in which 50 total rewards were earned, or until a total of 6000 (first problem) or 4000 (all other phases) rewards had been earned. Once this criterion was achieved with each pair individually, in the fifth and final phase (the final version of the task), the four pairs of objects were presented randomly intermixed across trials so that choice behavior had to be guided by the category membership of each object rather than a sequence of specific object choices. Training in this phase continued to the same criterion ( 2 consecutive sessions with a ratio of 2.94 or better or 4000 rewards earned, $\sim 80$ sessions of training). Choice behavior was above chance in the first session with intermixed problems, with a mean trials/reward ratio of 4.23 ; chance performance would be 16.3 (Gaffan et al., 2002). Monkeys that did not reach the 2.94 trials/reward criterion and advanced based on the cumulative number of rewards earned within a phase (ORB2, first problem; CON2, third problem and final phase; CON4, final phase) performed comparably in their preoperative performance test to other monkeys that had achieved the criterion during training. For all seven monkeys, the mean number of sessions required to complete all five phases of training was 188.4 (range, 80414); to complete the final phase of training, it was 46 (range, 11-149).

Object-in-place scene learning. The object-in-place scene learning task 
was adapted from Gaffan (1994). This task used artificially constructed background scenes that occupied the entire area of the display screen. The background scenes were generated by an algorithm based on a random number generator. Each scene was unique in that it varied in several randomly selected attributes including (1) the background color of the screen; (2) the location of ellipses on the screen; (3) the color, size, and orientation of ellipse segments; (4) the typographic character, clearly distinct in size from the foreground objects; and (5) the color of the typographic character. All the colors were assigned with the constraint that the foreground objects should be visible (i.e., there was a minimum separation in color space between the colors of a foreground object and the color of any element of its local background). Two background objects, small randomly chosen and colored typographic characters, were placed within each scene. In each scene, one of the two foreground objects was the correct one for the monkey to touch (rewarded), and the other was incorrect (unrewarded). The locations and identities of the foreground objects were fixed within each scene but varied between scenes. Because these scenes were randomly generated, an infinite number of unique scenes could be presented. For example stimuli, see Browning et al. (2005), Gaffan (1994), and supplemental Figure 2 (available at www.jneurosci.org as supplemental material). After each monkey learned to touch single foreground objects against a black background, additional scene elements were introduced in shaping programs until the monkey reliably touched the foreground object when presented with a new scene. Problems were then introduced with two foreground objects (one correct and one incorrect, as described above), and the number of scenes given in each session was gradually increased, based on each monkey's performance. Training continued until performance was stable (for all seven monkeys: mean, 59.1 sessions; range, 14-111).

In the final version of the task, 20 new scenes were presented in each session; the list of 20 scenes was repeated eight times. Each trial began with the presentation of a scene problem on the screen (a background scene containing two foreground objects). A touch to the correct object caused the object to flash for $2.4 \mathrm{~s}$, then the screen blanked, and a reward pellet (190 mg; P. J. Noyes) was delivered, followed by a $5 \mathrm{~s}$ intertrial interval. A touch to the incorrect object caused the screen to blank immediately, followed by a $20 \mathrm{~s}$ intertrial interval. Touches anywhere else in the scene caused the screen to blank, and the trial was repeated, after a $20 \mathrm{~s}$ intertrial interval. For the first repetition of the list of scenes only, incorrect responses were followed by a correction trial in which the scene was represented with only the correct object present. The subsequent seven repetitions of the list of scenes did not contain correction trials, and the scenes were presented in the same order in which they were encountered in the first run through the list. Monkeys learned which object in each scene was correct by trial and error, generally very rapidly during the first run through the list, because error rates were very low during the second run through the list (9-21.5\%; chance is 50\%). When the monkey completed the final trial of a session, the lunchbox opened, and the monkey received the large food reward. If the final trial was incorrect, a correction trial was given so that the monkey only ever received the large food reward after a correct response. The dependent measure was the number of errors (initial touches of the incorrect foreground object) in each presentation of the list of 20 scenes.

Performance tests. After completion of training on the scene learning and strategy tasks, all monkeys were given a preoperative performance test consisting of 24 sessions. The first session was scene learning, followed by five cycles of two sessions of strategy performance, followed by two sessions of scene learning, then two sessions of strategy performance, then a final session of scene learning. The sequence of sessions was thus STTSSTTSSTTSSTTSSTTSSTTS, where "S" represents a session of scene learning and " $\mathrm{T}$ " represents a session of strategy implementation testing. Data from the first 4 sessions were not considered, leaving 20 sessions of performance data ( 10 of scene learning, 10 of strategy implementation). The tasks were presented in their "final versions" as described above during this performance test. In this double-alternation design, we could compare performance on each task when it was preceded by performance on the same or a different task, although we did not observe any systematic variation in performance related to this variable either before or after surgery. This test was repeated in the same way beginning at least 2 weeks after surgery (for monkeys in the orbital group) or an equivalent period of rest for control monkeys.

Surgery. Neurosurgical procedures were performed in a dedicated operating theater under aseptic conditions. Each operated monkey's neurosurgical procedure consisted of a bilateral ablation of the orbital prefrontal cortex. In cases ORB2 and ORB3, steroids $(20 \mathrm{mg} / \mathrm{kg}$ methylprednisolone) were given intramuscularly the night before surgery, and three doses were given 4-6 h apart (intravenously or intramuscularly) on the day of surgery, to protect against intraoperative edema and postoperative inflammation. Each monkey was sedated on the morning of surgery with both ketamine $(10 \mathrm{mg} / \mathrm{kg}$, i.m. $)$ and xylazine $(0.5 \mathrm{mg} / \mathrm{kg}$, i.m.). Once sedated, the monkey was given atropine $(0.05$ $\mathrm{mg} / \mathrm{kg}$ ) to reduce secretions, antibiotic $(8.75 \mathrm{mg} / \mathrm{kg}$ amoxicillin) for prophylaxis of infection, opioid $(0.01 \mathrm{mg} / \mathrm{kg}$ buprenorphine, i.v.; repeated twice at $4-6 \mathrm{~h}$ intervals on the day of surgery, intravenously or intramuscularly) and nonsteroidal anti-inflammatory (either $0.2 \mathrm{mg} / \mathrm{kg}$ meloxicam, i.v., or $4 \mathrm{mg} / \mathrm{kg}$ carprofen, i.m.) agents for analgesia, and an $\mathrm{H} 2$ receptor antagonist ( $1 \mathrm{mg} / \mathrm{kg}$ ranitidine, i.v.) to protect against gastric ulceration as a side effect of the combination of steroid and nonsteroidal anti-inflammatory treatment. The head was shaved, and an intravenous cannula was put in place for intraoperative delivery of fluids (warmed sterile saline drip, $5 \mathrm{ml} / \mathrm{kg} / \mathrm{h}$ ). The monkey was moved into the operating theater, intubated, placed on isoflurane (ORB1 and ORB2, 1-2\%, to effect, in $100 \%$ oxygen) or sevoflurane (ORB3, 2.5-4.75\%, to effect, in $100 \%$ oxygen) anesthesia, and mechanically ventilated. Adjustable heating blankets allowed maintenance of normal body temperature during surgery. Heart rate, oxygen saturation of hemoglobin, mean arterial blood pressure, end tidal $\mathrm{CO}_{2}$, body temperature, and respiration rate were monitored continuously throughout surgery.

The monkey was placed in a head-holder, and the head was cleaned with alternating antimicrobial scrub and alcohol and draped to allow a midline incision. The skin and underlying galea were opened in layers. The temporal muscles were retracted as necessary to expose the skull surface over the intended lesion site. A bone flap was turned over the frontal lobes, and the craniotomy was extended with rongeurs as necessary. The dura was cut and reflected over the frontal lobes. The orbital prefrontal cortex was removed bilaterally extending from the fundus of the lateral orbital sulcus to the fundus of the rostral sulcus. The anterior and posterior limits were lines joining the tips of the lateral and medial orbital sulci, extending medially to the midline. All of the cortex was removed within these limits, including both banks of the medial orbital sulcus. Cortical tissue was removed by subpial aspiration using a small-gauge sucker insulated everywhere except at the tip; electrocautery was applied to remove the pia mater and control bleeding encountered during the ablation.

When the lesion was complete, the dura was sewn over the lesion site, the bone flap was replaced and held with loose sutures, and the skin and galea were closed in layers. The monkey was removed from the headholder, and anesthesia was discontinued. The monkey was extubated when a swallowing reflex was observed, returned to the home cage, and monitored continuously until normal posture was regained (usually within $10 \mathrm{~min})$. Nonsteroidal anti-inflammatory analgesic $(0.2 \mathrm{mg} / \mathrm{kg}$ meloxicam, orally) and antibiotic $(8.75 \mathrm{mg} / \mathrm{kg}$ amoxicillin, orally) treatment continued after surgery in consultation with veterinary staff, typically for $5 \mathrm{~d}$. Operated monkeys rejoined their social groups as soon as practicable after surgery, usually within $3 \mathrm{~d}$ of the operation.

Object-reward association learning. After the completion of the postoperative performance test on strategy and scene learning, monkeys were given three sets of 10 concurrent object-reward association learning problems; each set was trained to criterion before the next was presented. Each set comprised 10 pairs of clip-art objects, with each pair constituting a problem, and one of the two clip-art objects was arbitrarily designated correct in each pair. The objects were presented against a gray background, one on the left side of the screen and one on the right, which was randomized across trials. Touching the correct object resulted in the incorrect object disappearing, delivery of a reward pellet, and the correct object disappearing after $1 \mathrm{~s}$. Touching the incorrect object caused both objects to disappear immediately, and no reward was delivered. The intertrial interval was $5 \mathrm{~s}$ after a correct choice and $10 \mathrm{~s}$ after an incorrect one, and a touch to the screen during the intertrial interval reset it. Each 
Intended

$+36$

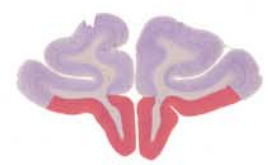

$+32$

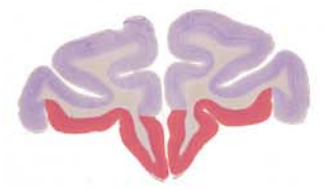

$+28$

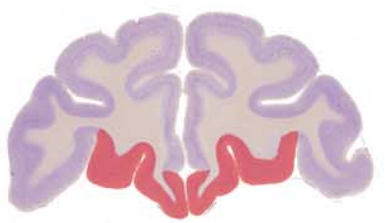

ORB1
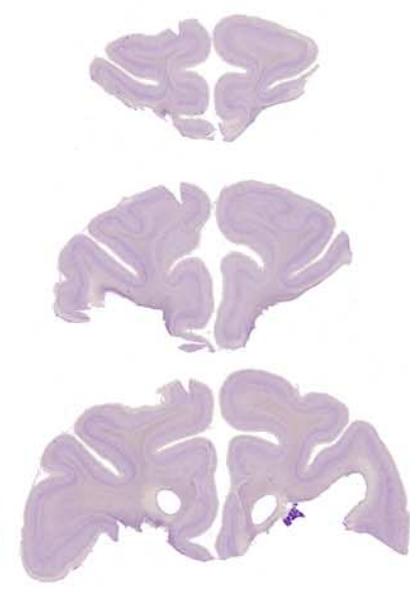

ORB2
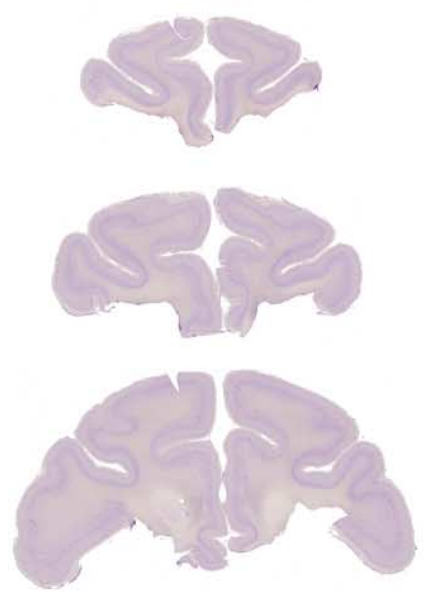

ORB3
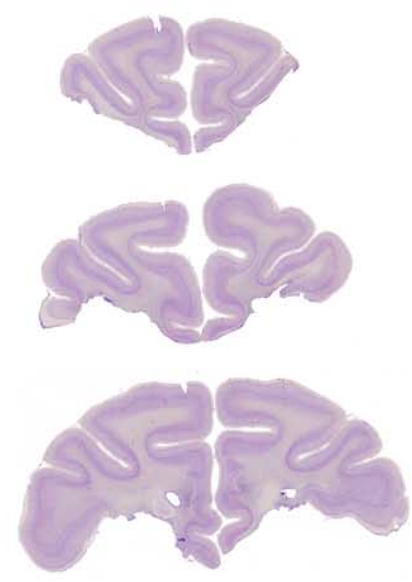

Figure 1. Lesions of orbital prefrontal cortex. The first column shows the extent of intended damage (red) on sections from the brain of a monkey without damage to orbital prefrontal cortex. The three remaining columns show histological sections from each of the three cases with orbital prefrontal lesions. Each row represents one approximate stereotaxic level, in millimeters anterior to the interaural plane, from anterior to posterior.

problem appeared once in each block of 10 trials. Ten blocks of trials were given in each test session. After the 10th block, the lunchbox opened and the large food reward was delivered. In the event that the last response of the session (on the 100th trial) was incorrect, another block began, and the session ended (and the lunchbox was delivered) after the first correct response in this block. Training on each set continued until a criterion of $90 \%$ correct responses in a single session was achieved, with a minimum of two sessions with each problem set.

Histology. After completion of behavioral training, each monkey was sedated with ketamine $(10 \mathrm{mg} / \mathrm{kg})$, deeply anesthetized with intravenous barbiturate, and transcardially perfused with $0.9 \%$ saline followed by $10 \%$ formalin. The brain was cryoprotected in formalin-sucrose and sectioned coronally on a freezing microtome at $50 \mu \mathrm{m}$ thickness. A 1 -in-10 series of sections through the area of the lesion was mounted on gelatin-coated glass microscope slides and stained with cresyl violet. Lesions are shown in Figure 1. The extent of removal of orbital prefrontal cortex was similar in all three cases and was essentially as intended. The caudolateral extent of the lesion was slightly greater in case ORB1 relative to the other two cases. Because of the variability in sulcal patterns on the orbital surface (Chiavaras and Petrides, 2000) and the similarity in lesion extent across the three cases, we did not attempt additional quantitative analysis of the extent of orbital prefrontal damage.

\section{Results}

\section{Strategy implementation}

Changes in performance between preoperative and postoperative testing were again analyzed by repeated-measures ANOVA with testing phase (preoperative vs postoperative) as a within-subject factor and lesion group (control or orbital prefrontal lesion) as a between-subjects factor. Bilateral ablation of orbital prefrontal cortex was completely without effect on strategy implementation performance, measured by the trials/reward ratio, which was unchanged after the orbital prefrontal lesion. There were no effects of lesion group, test phase, or test phase $\times$ group interaction $(F<$ $1)$. These data are plotted in Figure 2. The stability of performance between preoperative and postoperative assessments in both groups is evident.

\section{Scene learning}

Changes in performance between preoperative and postoperative testing were analyzed by repeated-measures ANOVA with testing phase (preoperative vs postoperative) and each trial (repetition) of the list of scenes as within-subject factors and lesion group

\section{Strategy Implementation}

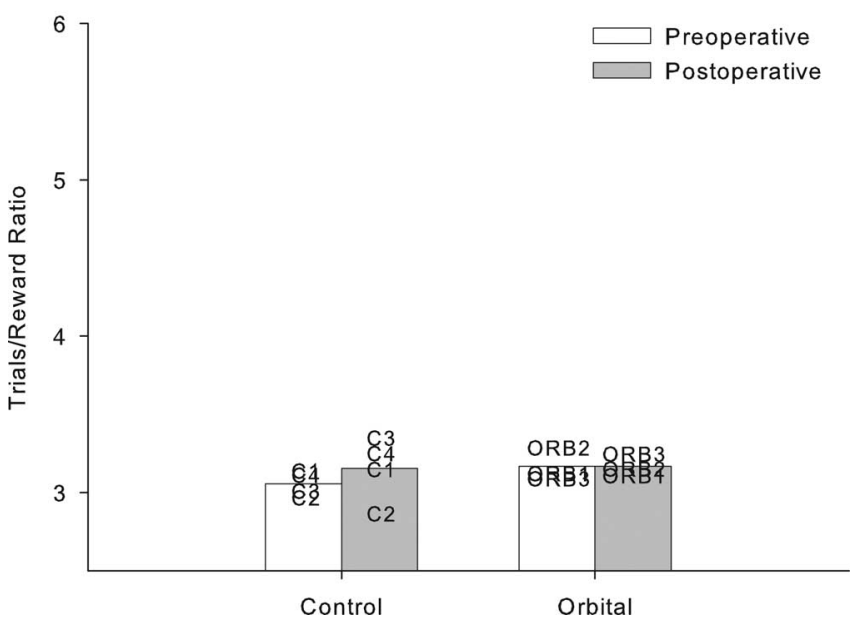

Figure 2. Individual subject performance in strategy implementation. Both control monkeys and monkeys with orbital prefrontal lesions perform comparably on preoperative and postoperative tests of strategy implementation performance.

(control or orbital prefrontal lesion) as a between-subjects factor. Bilateral ablation of orbital prefrontal cortex produced a significant impairment in object-in-place scene learning, with operated monkeys committing $\sim 2.5$ times as many errors postoperatively compared with control monkeys whose error rates were similar before and after a period of rest equivalent in time to postoperative recovery for the operated group. This analysis revealed a main effect of trial, as expected $\left(F_{(7,35)}=150.32 ; p<0.0005\right)$; a main effect of test phase $\left(F_{(1,5)}=8.76 ; p<0.0005\right)$; and a test phase by trial interaction $\left(F_{(7,35)}=6.90 ; p<0.0005\right)$. These latter two effects were driven by the performance of the orbital monkeys, because they were not present when data from the controls was analyzed alone $(F<1)$. Additionally, there were interactions of test phase with lesion group $\left(F_{(1,5)}=9.77 ; p<0.0005\right)$ and of test phase, trial, and lesion group $\left(F_{(7,35)}=5.64 ; p<0.0005\right)$. These data are plotted in Figures 3 and 4. Figure 3 shows learning curves across the eight repetitions of lists of 20 new scenes, which illustrate slower postoperative learning in the orbital group. Fig- 
Object-in-place Scene Learning
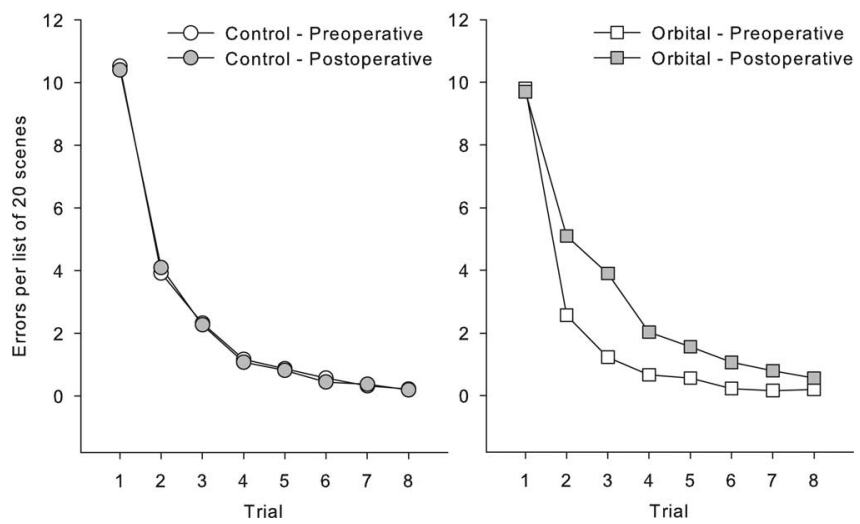

Figure 3. Performance on scene learning. Mean data for each group are shown in preoperative and postoperative performance, in errors per list of 20 scenes (on the vertical axis) on each of eight repetitions of each list of problems (horizontal axis). Performance is identical in control monkeys in preoperative and postoperative performance tests, but monkeys with orbital prefrontal lesions show a learning deficit postoperatively.

\section{Object-in-place Scene Learning}

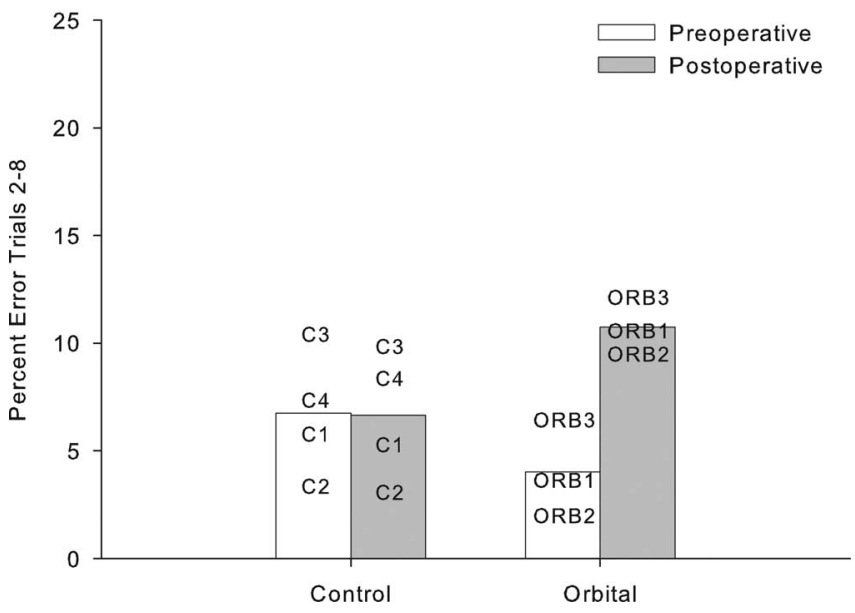

Figure 4. Individual subject data in scene learning. The dependent measure is the mean percentage error on trials 2- 8 of each list of new scenes (performance in trial 1 is at chance for each list, because it is the first time monkeys have encountered the scenes, and they must discover the rewarded object by trial and error). The four control monkeys show stable performance between preoperative and postoperative performance tests; each monkey with an orbital prefrontal lesion is impaired relative to its preoperative performance.

ure 4 shows a summary measure (percentage of errors on trials 2-8 of each new list of scenes) for each monkey preoperatively and postoperatively and demonstrates that each orbital monkey makes more errors postoperatively, whereas performance of the control monkeys is stable. A within-subjects (preoperative vs postoperative) comparison of the summary measure of the number of errors on trials $2-8$ for the orbital group revealed an identical result $\left(t_{(2)}=12.75 ; p=0.006\right)$.

We considered whether poor postoperative performance in the orbital group could be attributed to perseveration of initial incorrect responses. By this view, an involvement of the orbital prefrontal cortex in behavioral flexibility could impair scene learning because monkeys continue emitting initial incorrect responses and do not change them in response to feedback. This would predict that performance would be more impaired for scenes in which the initial response was incorrect compared with
Object-in-place Scene Learning

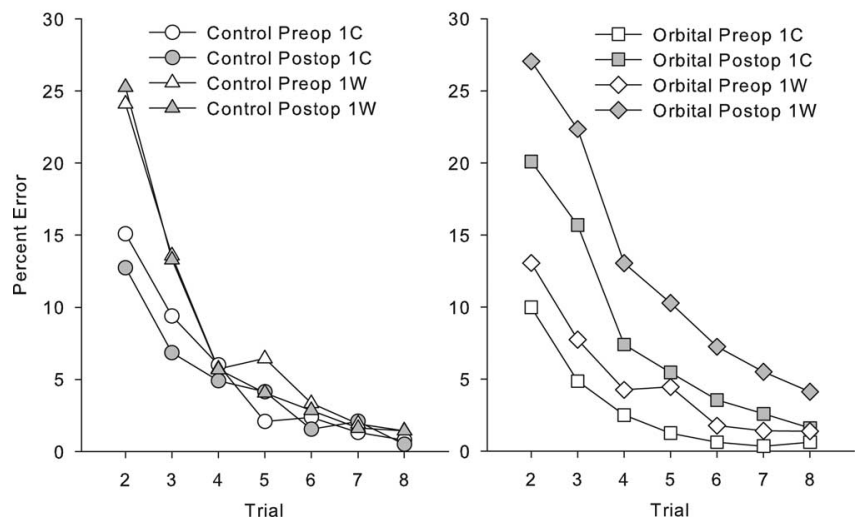

Figure 5. Performance on scene learning divided by whether the initial response to each scene was correct (1C) or incorrect (1W). Monkeys make more errors throughout learning on 1W scenes compared with $1 C$ scenes. Monkeys with orbital prefrontal lesions, importantly, make more errors on both $1 \mathrm{C}$ scenes and $1 \mathrm{~W}$ scenes postoperatively (compare open symbols with shaded symbols). Thus, their impairment is not limited to problems in which they must adjust their responding after an error.

scenes in which the initial response was correct. However, if trials are subdivided based on whether the initial response to each scene (during its first presentation in the session) is correct (1C) or wrong $(1 \mathrm{~W})$, there is no differential effect of lesion on trials in which the first response to the scene is wrong relative to trials on which the first response to the scene is correct. Comparison of responding between preoperative and postoperative revealed expected main effects of trial, test phase (preoperative/postoperative), $1 \mathrm{C} / 1 \mathrm{~W}$, and group, as well as an interaction of $1 \mathrm{C} / 1 \mathrm{~W}$ with trial but no interaction of $1 \mathrm{C} / 1 \mathrm{~W}$ with group $\left(F_{(1,5)}=0.275 ; p=0.62\right)$; threeway interaction of $1 \mathrm{C} / 1 \mathrm{~W}$, test phase, and group $\left(F_{(1,5)}=4.71 ; p=\right.$ $0.08)$; or four-way interaction of $1 \mathrm{C} / 1 \mathrm{~W}$, test phase, group, and trial $\left(F_{(6,30)}=0.597 ; p=0.73\right)$. These data are plotted in Figure 5. Although the three-way interaction of $1 \mathrm{C} / 1 \mathrm{~W}$, test phase, and group is marginal, test phase $\times$ group interactions are present when $1 \mathrm{C}$ and $1 \mathrm{~W}$ trials are considered separately $\left(F_{(1,5)}=61.79\right.$ and 70.32, respectively; $p \leq 0.001$. Thus, poor performance after the orbital prefrontal lesion cannot be explained entirely by an increased perseverative tendency to continue to respond incorrectly to scenes where the initial response is incorrect. Instead, monkeys with orbital damage are impaired in learning whether they receive positive or negative feedback from their initial choices.

\section{Object-reward association learning}

There were no differences between the groups in the mean number of errors to criterion, averaged across the three sets: control: mean, 53.17; range, 24-93.33; orbital: mean, 71.67; range, 32$\left.141.3 ; t_{(5)}=0.54 ; p=0.61\right)$.

\section{Discussion}

The present study makes two contributions to our understanding of the functions of the orbital prefrontal cortex. First, we identify a specific involvement of this region of prefrontal cortex in object-in-place scene learning, a monkey model of episodic memory formation. Second, surprisingly, the same monkeys were not impaired in performance of a preoperatively learned strategy implementation task that requires continuously adjusting choice behavior of objects associated with different reward schedules. Both of these tasks require frontal-inferotemporal interaction (Gaffan et al., 2002; Browning et al., 2005). 


\section{Scene learning}

It is not obvious that the impairment in scene learning after orbital lesions can be explained by a failure to adjust initial responses as a consequence of reward feedback, a deficit in flexible stimulus-reward or stimulus-action associations, or a failure of associative learning generally. Monkeys with orbital prefrontal lesions learned new sets of object-reward association problems as efficiently as unoperated controls, so their associative learning ability was intact. There was also no evidence that their impairment in scene learning was restricted to problems in which their first response was an error (1W trials), which would be necessary to support the hypothesis that their impairment was one of inflexibility or insensitivity to feedback in the form of failing to receive reward for their initial choice. Instead, learning was impaired whether the initial response was correct or incorrect. This is consistent with recent data from selective lesions of orbital prefrontal cortex on reversal learning, which result in errors in the learning phase of reversed discriminations but do not cause perseverative behavior per se (Izquierdo et al., 2004).

The impairment in scene learning after orbital prefrontal lesions appears milder than that which follows disconnection of ventrolateral prefrontal cortex from inferotemporal cortex (Wilson et al., 2007). This may suggest that ventrolateral prefrontal cortex is more critical to scene learning than orbital prefrontal cortex, but monkeys in the present study had better preoperative scene learning ability than monkeys in the study of ventrolateral lesions (Wilson et al., 2007), complicating a direct comparison of levels of postoperative performance. Furthermore, differences in lesion methodology between the two studies (disconnection of ventrolateral prefrontal cortex and inferotemporal cortex in the previous study vs bilateral orbital prefrontal lesions in the present study) also make a straightforward comparison of the severity of deficits difficult.

Activity within human orbitofrontal cortex is associated with demands on memory encoding (Frey and Petrides, 2000, 2002; Petrides et al., 2002). This may be associated with noticing novel information and facilitating its encoding in connected inferotemporal areas (Frey and Petrides, 2002). Additionally, this region may be involved in strategic processes in memory encoding, because orbitofrontal activation is associated with "judgments of learning," assessments by the participant of the likelihood whether an experience will be remembered or not (Kao et al., 2005). Thus, the mild impairment caused by orbital prefrontal lesions in the present study may represent a contribution of this cortical area to strategies used by the monkeys to maximize learning in the task or to allocate cognitive resources to scene memory. This would be congruent with a hypothesized role for prefrontal cortex in "top-down" control of memory processing resources (Dove et al., 2006) and with the involvement of mediodorsal thalamus in scene learning (Gaffan and Parker, 2000; Mitchell et al., 2007).

\section{Strategy implementation}

The lack of impairment in strategy implementation performance after orbital prefrontal lesions was surprising. Neurons in orbital prefrontal cortex reflect proximity to reward as the monkey advances through a reward schedule (Ichihara-Takeda and Funahashi, 2006; Simmons and Richmond, 2007) as well as behavior-guiding rules (Wallis et al., 2001) that would be expected to be critical for performance of this strategy implementation task. As already noted, damage to orbital prefrontal cortex is associated with cognitive inflexibility and impaired reversal learning (Jones and Mishkin, 1972; Dias et al., 1996; McAlonan and Brown, 2003; Hornak et al., 2004), although not necessarily perseverative behavior in reversal learning (Izquierdo et al., 2004). Orbital prefrontal lesions do induce perse- verative behavior in acquisition of secondary reinforcement schedules (Pears et al., 2003) and extinction (Izquierdo and Murray, 2005). Monkeys must switch repeatedly between two different categories of objects to earn rewards efficiently in the strategy implementation task and must avoid responding to the relatively attractive sporadic objects, which lead immediately to reward when chosen appropriately, while making chains of persistent responses that do not. Thus, inflexible stimulus-reward associations after orbital prefrontal damage might be expected to disrupt performance, because this task is guided by reward outcomes of object choices. Moreover, the monkeys in the current study were also impaired in another test of decision-making and choice behavior, the reinforcer devaluation task (our unpublished observation), that has been shown to be sensitive to orbital prefrontal damage before (Izquierdo et al., 2004). Thus, they have impairments in decision-making and choice behavior but not in the setting of the strategy implementation task.

It is possible that performance of this task (making a fixed number of choices from one object category, followed by a single choice from the other once reward is delivered) becomes habitual, so that performance does not reflect application of strategies or rules at all, but rather a complex visual habit. On this view, performance of the task could occur without cortical involvement at all, explaining the lack of effect of orbital prefrontal damage in the present study. However intrahemispheric frontal-inferotemporal cortical interaction is required for performance of this task (Gaffan et al., 2002). The prefrontal regions outside orbital prefrontal cortex that are required for this task remain to be identified. Because choice behavior in the task requires selection of different visual stimuli at different points in the sequence, a fixed sequence of particular object choices cannot be learned, so this task does not resemble classical tests of habit in monkeys, such as visual discrimination learning with long intertrial intervals in which particular objects are consistently associated with reward or nonreward (Malamut et al., 1984; Fernandez-Ruiz et al., 2001). The extent to which responding in the strategy implementation task is based on habit versus representation of the goal remains to be determined; modification of the task such that different strategies lead to different reinforcers could help address this question.

Some aspects of cognitive flexibility and behavioral inhibition of reward-related behavior survive ablation of orbital prefrontal cortex. Monkeys with damage to this region learn a reversed reward contingency task as efficiently as controls, in which they are offered a choice between a small and large reward but are given the reward opposite to the one they choose. Thus, they must avoid choosing the large reward in favor of the small one, to obtain the large reward (Chudasama et al., 2007). A generalized deficit in inhibitory control or of behavioral inflexibility would predict an impairment, or complete failure, in mastering this task. Notably, this learning took place postoperatively, so the lack of impairment cannot be ascribed to the retention and successful application of behavioral rules that were acquired preoperatively. These findings, together with the present ones, place limits on the generality of the involvement of orbital prefrontal cortex in cognitive flexibility and strategic behavior related to stimulus-reward associations. They suggest that, perhaps given the presence of a few well defined rules that govern reward delivery, monkeys with orbital prefrontal damage can apply these rules effectively to guide their behavior, in these instances to maximize the amount of reward (Chudasama et al., 2007) or rate of reward delivery (present study).

An alternative view of the strategy implementation task is that it may share more in common with tests of behavioral flexibility related to the use of stimulus attributes or behavioral strategies for discrimination learning (attentional set) that are thought to engage frontal regions outside the orbital prefrontal cortex (Dias 
et al., 1996; Ragozzino et al., 1999; Rich and Shapiro, 2007). Thus, the strategy implementation task may place a greater demand on higher-order representations of categorical or dimensional information represented outside orbital prefrontal cortex, rather than the use of reward feedback and the flexible selection of different categories of objects associated with different reward schedules. Although it remains surprising in the context of known effects of orbital prefrontal lesions that performance of this task is unaffected by these lesions, the present data may also suggest that prefrontal regions outside of orbital prefrontal cortex play a greater role in representing reward-related aspects of tasks when the task requires the representation of categorical or dimensional information in these extra-orbital prefrontal regions. This would be consistent with electrophysiological recording studies that find representation of, for example, both reward and response information in dorsolateral prefrontal cortex (Leon and Shadlen, 1999; Wallis and Miller, 2003). A highly speculative conclusion from the current data are that if other prefrontal regions must be engaged to represent task-relevant information that cannot be represented in the orbital prefrontal cortex (e.g., information related to category membership), then the orbital prefrontal cortex is no longer required for the representation of reward-related information that is critical to task performance once it is learned, so that orbital lesions do not impair behavior.

\section{References}

Aggleton JP, McMackin D, Carpenter K, Hornak J, Kapur N, Halpin S, Wiles CM, Kamel H, Brennan P, Carton S, Gaffan D (2000) Differential cognitive effects of colloid cysts in the third ventricle that spare or compromise the fornix. Brain 123:800-815.

Baxter MG, Gaffan D (2007) Asymmetry of attentional set in rhesus monkeys learning colour and shape discriminations. Q J Exp Psychol 60:1-8.

Browning PG, Easton A, Buckley MJ, Gaffan D (2005) The role of prefrontal cortex in object-in-place learning in monkeys. Eur J Neurosci 22:3281-3291.

Carmichael ST, Price JL (1995) Limbic connections of the orbital and medial prefrontal cortex in macaque monkeys. J Comp Neurol 363:615-641.

Chiavaras MM, Petrides M (2000) Orbitofrontal sulci of the human and macaque monkey brain. J Comp Neurol 422:35-54.

Chudasama Y, Kralik J, Murray E (2007) Rhesus monkeys with orbital prefrontal cortex lesions can learn to inhibit prepotent responses in the reversed reward contingency task. Cereb Cortex 17:1154-1159.

Dias R, Robbins TW, Roberts AC (1996) Dissociation in prefrontal cortex of affective and attentional shifts. Nature 380:69-72.

Dove A, Brett M, Cusack R, Owen AM (2006) Dissociable contributions of the mid-ventrolateral frontal cortex and the medial temporal lobe to human memory. NeuroImage 31:1790-1801.

Fellows LK, Farah MJ (2003) Ventromedial frontal cortex mediates affective shifting in humans: evidence from a reversal learning paradigm. Brain 126:1830-1837.

Fernandez-Ruiz J, Wang J, Aigner TG, Mishkin M (2001) Visual habit formation in monkeys with neurotoxic lesions of the ventrocaudal neostriatum. Proc Natl Acad Sci USA 98:4196-4201.

Frey S, Petrides M (2000) Orbitofrontal cortex: a key prefrontal region for encoding information. Proc Natl Acad Sci USA 97:8723-8727.

Frey S, Petrides M (2002) Orbitofrontal cortex and memory formation. Neuron 36:171-176.

Gaffan D (1994) Scene-specific memory for objects: a model of episodic memory impairment in monkeys with fornix transection. J Cogn Neurosci 6:305-320.

Gaffan D, Parker A (1996) Interaction of perirhinal cortex with the fornixfimbria: memory for objects and "object-in-place" memory. J Neurosci 16:5864-5869.

Gaffan D, Parker A (2000) Mediodorsal thalamic function in scene memory in rhesus monkeys. Brain 123:816-827.

Gaffan D, Easton A, Parker A (2002) Interaction of inferior temporal cortex with frontal cortex and basal forebrain: double dissociation in strategy implementation and associative learning. J Neurosci 22:7288-7296.

Hornak J, O’Doherty J, Bramham J, Rolls ET, Morris RG, Bullock PR, Polkey
CE (2004) Reward-related reversal learning after surgical excisions in orbito-frontal or dorsolateral prefrontal cortex in humans. J Cogn Neurosci 16:463-478.

Ichihara-Takeda S, Funahashi S (2006) Reward-period activity in primate dorsolateral prefrontal and orbitofrontal neurons is affected by reward schedules. J Cogn Neurosci 18:212-226.

Izquierdo A, Murray EA (2005) Opposing effects of amygdala and orbital prefrontal cortex lesions on the extinction of instrumental responding in macaque monkeys. Eur J Neurosci 22:2341-2346.

Izquierdo A, Suda RK, Murray EA (2004) Bilateral orbital prefrontal cortex lesions in rhesus monkeys disrupt choices guided by both reward value and reward contingency. J Neurosci 24:7540-7548.

Jones B, Mishkin M (1972) Limbic lesions and the problem of stimulusreinforcement associations. Exp Neurol 36:362-377.

Kao YC, Davis ES, Gabrieli JD (2005) Neural correlates of actual and predicted memory formation. Nat Neurosci 8:1776-1783.

Kondo H, Saleem KS, Price JL (2005) Differential connections of the perirhinal and parahippocampal cortex with the orbital and medial prefrontal networks in macaque monkeys. J Comp Neurol 493:479-509.

Leon MI, Shadlen MN (1999) Effect of expected reward magnitude on the response of neurons in the dorsolateral prefrontal cortex of the macaque. Neuron 24:415-425.

Malamut BL, Saunders RC, Mishkin M (1984) Monkeys with combined amygdalo-hippocampal lesions succeed in object discrimination learning despite 24-hour intertrial intervals. Behav Neurosci 98:759-769.

McAlonan K, Brown VJ (2003) Orbital prefrontal cortex mediates reversal learning and not attentional set shifting in the rat. Behav Brain Res 146:97-103.

Meunier M, Bachevalier J, Mishkin M (1997) Effects of orbital frontal and anterior cingulate lesions on object and spatial memory in rhesus monkeys. Neuropsychologia 35:999-1015.

Mitchell AS, Baxter MG, Gaffan D (2007) Dissociable performance on scene learning and strategy implementation after lesions to magnocellular mediodorsal thalamic nucleus. J Neurosci, in press.

Parker A, Gaffan D (1997) Mammilary body lesions in monkeys impair object-in-place memory: functional unity of the fornix-mamillary system. J Cogn Neurosci 9:512-521.

Pears A, Parkinson JA, Hopewell L, Everitt BJ, Roberts AC (2003) Lesions of the orbitofrontal but not medial prefrontal cortex disrupt conditioned reinforcement in primates. J Neurosci 23:11189-11201.

Petrides M, Alivisatos B, Frey S (2002) Differential activation of the human orbital, mid-ventrolateral, and mid-dorsolateral prefrontal cortex during the processing of visual stimuli. Proc Natl Acad Sci USA 99:5649-5654.

Ragozzino ME, Wilcox C, Raso M, Kesner RP (1999) Involvement of rodent prefrontal cortex subregions in strategy switching. Behav Neurosci 113:32-41.

Remijnse PL, Nielen MM, Uylings HB, Veltman DJ (2005) Neural correlates of a reversal learning task with an affectively neutral baseline: an eventrelated fMRI study. NeuroImage 26:609-618.

Rich EL, Shapiro ML (2007) Prelimbic/infralimbic inactivation impairs memory for multiple task switches, but not flexible selection of familiar tasks. J Neurosci 27:4747-4755.

Roesch MR, Olson CR (2004) Neuronal activity related to reward value and motivation in primate frontal cortex. Science 304:307-310.

Simmons JM, Richmond BJ (2007) Dynamic changes in representations of preceding and upcoming reward in monkey orbitofrontal cortex. Cereb Cortex, in press.

Wallis JD, Miller EK (2003) Neuronal activity in primate dorsolateral and orbital prefrontal cortex during performance of a reward preference task. Eur J Neurosci 18:2069-2081.

Wallis JD, Anderson KC, Miller EK (2001) Single neurons in prefrontal cortex encode abstract rules. Nature 411:953-956.

Watanabe M (1996) Reward expectancy in primate prefrontal neurons. Nature 382:629-632.

Webster MJ, Bachevalier J, Ungerleider LG (1994) Connections of inferior temporal areas TEO and TE with parietal and frontal cortex in macaque monkeys. Cereb Cortex 4:470-483.

Wilson CRE, Gaffan D, Mitchell AS, Baxter MG (2007) Neurotoxic lesions of ventrolateral prefrontal cortex impair object-in-place scene memory. Eur J Neurosci 25:2514-2522. 